\title{
Eigenständiges Fach Notfallmedizin nicht im Sinne des schweizerischen Gesundheitswesens
}

\author{
Georg Schulthess, \\ Jean-Michel Gaspoz ${ }^{a}$, \\ Ralph A. Schmid ${ }^{b}$ \\ Andreas Platz', \\ Esther Bächlid, \\ Philipp M. Lenzlinger
}

a Präsident der Schweizerischen Gesellschaft für Allgemeine Innere Medizin (SGIM)

b Präsident der Schweizerischen Gesellschaft für Chirurgie (SGC)

c Präsident der Schweizerischen Gesellschaft für Allgemeinchirurgie und Traumatologie (SGACT)

d Präsidentin der Internistischen Chefärztevereinigung Schweiz (SGIM)
Korrespondenz:

Prof. Dr. med. Georg Schulthess Chefarzt Medizinische Klinik

Spital Männedorf

CH-8708 Männedorf

Dr. med. Philipp M. Lenzlinger Leitender Arzt Chirurgische

Klinik und Leiter Unfallchirurgie Spital Limmattal

CH-8952 Schlieren
Im Artikel «Länderübergreifende Zusammenarbeit in der Notfallmedizin» [1] wird das Schaffen eines eigenständigen Fachgebiets Notfallmedizin angeregt. Das neue Fachgebiet würde in eigenständigen Strukturen und mit eigenen Fachärzten betrieben. Die entsprechenden Facharztstellen müssten neu geschaffen werden, und die Fachärzte würden, wie vorgeschlagen, einer eigenen fünfjährigen Weiterbildung unterstehen.

Dieses Vorhaben entspräche einem tiefen, ungünstigen und erst noch unnötigen Einschnitt ins schweizerische Gesundheitssystem: Es würde ohne Notwendigkeit eine zusätzliche Segmentierung der medizinischen Versorgung geschaffen, dadurch erhebliche Mehrkosten verursacht und zudem nachteilig ins ärztliche Weiterbildungssystem eingegriffen. Paradoxerweise wird in dem genannten Artikel [1] das Schaffen des zusätzlichen Fachgebiets damit begründet, dass bereits aktuell die Segmentierung der Notfallversorgung in den Spitälern hoch sei und damit einem effizienten medizinischen Vorgehen entgegenstehe.

Die Argumentation für ein eigenständiges Fachgebiet Notfallmedizin erfolgt nicht im Sinne, sondern zuungunsten des schweizerischen Gesundheitswesens. Deshalb muss an dieser Stelle klar widersprochen werden:

\section{Notfallmedizin als unverzichtbarer Bestand- teil etablierter Fachgebiete}

- Die Krankheitsbilder der grossen Mehrheit der Notfallpatienten erfordern Wissen, Erfahrung und Fertigkeiten aus den Fachgebieten Allgemeine Innere Medizin und Chirurgie. Zudem wird eine Mehrheit der Patienten notfallmässig in die Spitäler aufgenommen - für die Innere Medizin sind es über 80\%. Auf der Notfallstation erfolgen Triage, Diagnostik und Erstellen des Behandlungsplans.

- Damit ist die Notfallmedizin ein natürlicher und unverzichtbarer Bestandteil der Allgemeinen Inneren Medizin und der Chirurgie. Es ist deshalb folgerichtig und nur im Sinne einer guten $\mathrm{Pa}$ tientenbetreuung, den klinischen Notfallbetrieb (im eigentlichen Sinn des Wortes) interdisziplinär zu betreiben, also in einer gutorganisierten Zusammenarbeit der involvierten Fachdisziplinen.

\section{Zusammenfassung}

Notfallmedizin ist ein inhärenter Bestandteil der Fachgebiete Allgemeine Innere Medizin und Chirurgie einschliesslich der Weiterbildung zum praktizierenden Arzt sowie zum Internisten oder Chirurgen im Spitalbetrieb. Diese fachliche Aufstellung ist Basis für die gute Integration einer Notfallstation in den Spitalbetrieb mit longitudinaler Patientenbetreuung. Das Etablieren der Notfallmedizin als eigenständiges Fachgebiet würde hingegen (I) eine zusätzliche, unnötige Segmentierung der medizinischen Versorgung schaffen, (II) nachteilig ins ärztliche Weiterbildungssystem eingreifen und (III) erhebliche Kosten verursachen.

\section{Notfallmedizin als inhärenter Bestandteil ärztlicher Weiterbildung}

- Dementsprechend ist die Notfallmedizin ein inhärenter Bestandteil der Weiterbildung zum praktizierenden Arzt (AIM) wie auch zum Internisten oder Chirurgen im Spitalbetrieb: Der Umgang mit leichten (wie Halsschmerzen) bis zu schwersten Notfallsituationen muss gelernt und regelmässig geübt werden. Es wäre klar zuungunsten des schweizerischen Gesundheitssystems, würde die Notfallmedizin unter Ausschluss angehender Hausärzte, Internisten und Chirurgen an fachfremde Notfallärzte ausgelagert.

\section{Unverzichtbarkeit der Allgemeininternisten und Chirurgen}

- Die zeitnahe Verfügbarkeit und direkte Involvierung internistischer respektive chirurgischer Fachärzte im Spital ist bei dringlichen, schweren Notfallsituationen unverzichtbar - je dringlicher und fachlich anspruchsvoller die Situation ist, umso mehr trifft dies zu.

- Die direkte Betreuung durch internistische oder chirurgische Fachärzte auf der Notfallstation ist auch bei nicht-dringlichen Patienten sinnvoll, da sich solche Patienten immer häufiger auf der Notfallstation einfinden und dort ohne Schnittstelle 
auf die entsprechenden Stationen aufgenommen werden sollen. Der Notfallstation kommt dabei die Funktion einer Aufnahmestation zu.

\section{Longitudinale Patientenbetreuung im Spital}

- Die selbstverständliche Integration einer Notfallstation in den Spitalbetrieb ermöglicht eine longitudinale Patientenbetreuung: Die notfallmässige Aufnahme eines Patienten ins Spital und die weitere Betreuung auf Station bis zur Entlassung wird, wenn immer möglich, in die Hände nur einer ärztlichen Einheit gelegt - einem integrierten Behandlungspfad folgend.

- Eine wie in dem Beitrag von U. Bürgi et al. [1] erwähnte starke Segmentierung der Notfallversorgung findet sich nur in wenigen Grossspitälern (wie USZ, CHUV), nicht aber in den anderen, mehrheitlich mittelgrossen und kleineren Spitälern der Schweiz. Hier erfolgt die medizinische Versorgung vorwiegend durch Allgemeininternisten und Chirurgen. Und es bestehen weder der fachliche Bedarf noch die finanziellen Mittel für den Aufbau eines zusätzlichen Dienstbetriebes mit rund um die Uhr präsenten/verfügbaren, fachlich eigenständigen Notfallmedizinern.

- Aber auch in den Grossspitälern besteht nicht der Bedarf nach fachlicher Eigenständigkeit, sondern nach einer gutorganisierten und sinnvoll in den Spitalbetrieb integrierten Notfallstation. Die fachliche Kompetenz ist durch die kontinuierliche Präsenz von Internisten und Chirurgen sowie die Verfügbarkeit von Fachärzten jeglicher Ausrichtungen und Spezialitäten kontinuierlich sichergestellt. wird durch diese Disziplin fachlich kompetent und effizient abgedeckt. Gleiches gilt für die Allgemeine Innere Medizin, deren Kerntätigkeit die Betreuung akut erkrankter polymorbider Patienten ist. Bei der Aufnahme solcher Patienten ins Spital ist es oft auch hilfreich, dass diese mit ihren zugrundeliegenden Problemen den internistischen Fachärzten schon von früheren Spitalaufenthalten her bekannt sind.

- Das Aufrechterhalten eines kontinuierlichen Dienstbetriebes mit Fachärzten in Notfallmedizin würde für die Spitäler einen grossen finanziellen Aufwand bedeuten. Ein solcher Dienstbetrieb wäre aber angesichts eines eigenständigen Fachgebiets Notfallmedizin wohl zwingend. Denn die Verantwortung für den Notfallbetrieb könnte nicht (auch nicht während der Nacht, Wochenenden oder Feiertagen) an Fachärzte übertragen werden, deren Fachgebiete und Weiterbildung die Notfallmedizin nicht (mehr) beinhalten würden.

Das schweizerische Gesundheitswesen gilt als weltweit eines der Besten, dies schliesst - als wesentlichen Faktor - die zeitnahe und kompetente Notfallversorgung der Bevölkerung mit ein. Diese Versorgung beinhaltet die Bereitschaft und Kompetenz der praktizierenden Ärzte, Notfallpatienten auch kurzfristig innerhalb ihrer Sprechstunden anzunehmen und zu betreuen. Diese Versorgung beruht im Spital auf einer guten Integration der Notfallstation in den klinischen Betrieb mit Fachverantwortung bei der Inneren Medizin und der Chirurgie. Damit können Schnittstellen vermieden, Fehlerquellen umgangen und eine ad-

«Es wäre klar zuungunsten des Gesundheitssystems, würde die Notfallmedizin unter Ausschluss angehender Hausärzte, Internisten und Chirurgen an fachfremde Notfallärzte ausgelagert.»

\section{Ein grosser, kontraproduktiver Aufwand}

- Ein überwiegender Anteil der Notfallmedizin betrifft die Grundversorgung, innerhalb welcher einfach strukturiertes Handeln aus fachlichen und wirtschaftlichen Gründen wesentlich ist. Das Etablieren eines Fachgebiets Notfallmedizin würde deshalb das Gesundheitswesen der Schweiz erheblich verkomplizieren und verteuern.

- Vor allem aber würde das neue Fachgebiet nicht eine Lücke im Gesundheitssystem schliessen, sondern müsste aus den Zuständigkeitsbereichen etablierter Fachgebiete ausgegliedert und abgegrenzt werden. Ein Fachgebiet wie beispielsweise die Traumatologie ist inhärenter Teil der Notfallmedizin, und das zugehörige Segment äquate ärztliche Weiterbildung ausgerichtet werden. Im Gegensatz dazu würde sich eine Monopolisierung des Notfallbetriebes mit eigenständigen Strukturen und eigenen Fachärzten zuungunsten des Schweizerischen Gesundheitssystems auswirken.

Unter dem Aspekt der medizinisch-fachlichen Qualität, der fachärztlichen Weiterbildung und der Wirtschaftlichkeit sind somit jegliche Bestrebungen, die Notfallmedizin aus den angestammten Fachgebieten herauszulösen, konsequent zurückzuweisen.

1 Bürgi U, Christ M, Hogan B, Behringer W, Dodt C. Länderübergreifende Zusammenarbeit in der Notfallmedizin. Schweiz Ärztezeitung. 2013;94(50):1899-1900. 